\title{
AF291 and AE391 antibodies recognize a Dictyostelium HA-tagged protein by Western blot
}

Tania Jauslin

\begin{abstract}
The AF291 and AE391 antibodies detect an HA-tagged Dictyostelium discoideum protein by Western blot.

\section{Introduction}

The HA tag is a commonly used epitope tag derived from the human influenza virus hemagglutinin protein. AF291 and AE391 have been shown to recognize an HA-tagged protein in HEK293T cells by Western blot (Keszei and Picard, 2019). Here, we describe the ability of AF291 and AE391 antibodies to recognize an HA-tagged $D$. discoideum protein by Western blot.
\end{abstract}

\section{Materials \& Methods}

\begin{abstract}
Antibodies: ABCD_AF291 and ABCD_AE391 (ABCD nomenclature, web.expasy.org/abcd/; Lima et al., 2019) were produced by the Geneva Antibody Facility (www.unige.ch/antibodies/) as mini-antibodies with the antigen-binding $\mathrm{scFv}$ fused to the $\mathrm{Fc}$ region of a mouse $\operatorname{IgG}$ (IgG2b for AF291 and IgG2a for AE391). The synthesized $\mathrm{scFv}$ sequences (GeneArt, Invitrogen) correspond to the sequences of the variable regions of the monoclonal anti-HA clones 12CA5 (for AF291, Niman et al., 1983) and 26/9 (for AE391, Churchill et al., 1994) joined by a peptide linker (GGGGS) 3 . HEK293 suspension cells (growing in FreeStyleTM 293 Expression Medium, Gibco \#12338) were transiently transfected with the vectors coding for each scFv-Fc. Supernatants (70 and 10 $\mathrm{mg} / \mathrm{L}$, respectively) were collected after 5 days.
\end{abstract}

Antigen: $D$. discoideum alyL $\mathrm{KO}$ cells were stably transfected with an expression vector derived from $\mathrm{pDXA}$ 3C expressing a C-terminally HA-tagged AlyA protein (Amoeba LYsozyme, DDB_G0275123, residues 1-138). The HA sequence used was YPYDVPDYA. alyA-KO cells were used as negative control, and anti-AlyA MRB376 antibody was used as positive control to confirm overexpression of the tagged protein (Lamrabet, 2019).

Protocol: $5 \times 10^{6}$ D. discoideum cells were pelleted and resuspended in $200 \mu \mathrm{L}$ of reducing sample buffer $(20.6 \%$ (w/v) sucrose, $100 \mathrm{mM}$ Tris $\mathrm{pH}$ 6.8, $10 \mathrm{mM}$ EDTA, 0.1\% $(\mathrm{w} / \mathrm{v})$ bromophenol blue, 4\% (w/v) SDS, 6\% (v/v) $\beta$ mercaptoethanol). $20 \mu \mathrm{L}$ of each sample was migrated (200 V, $30 \mathrm{~min}$ ) on a 4-20\% acrylamide gel (MiniPROTEAN ${ }^{\circ}$ TGX ${ }^{\mathrm{TM}}$ Precast Gel, Genscript \#00655), and transferred to a nitrocellulose membrane using a dry transfer system for 7 minutes (iBlot gel transfer device, Invitrogen \#IB23001). The membranes were blocked during 2 hours in PBS containing $0.1 \%(\mathrm{v} / \mathrm{v})$ Tween 20 and $7 \%(\mathrm{w} / \mathrm{v})$ milk, and washed three times for 5 minutes in PBS $+0.1 \%(\mathrm{v} / \mathrm{v})$ Tween 20 . The membranes were then incubated with the different antibodies (non-diluted, dilution 1:10, and 1:2 in PBS-Tween for AE391, AF291, and MRB376, respectively), for 90 minutes at room temperature and washed three times for 5 minutes. The membranes were then incubated with horseradish peroxidase-coupled goat anti-mouse IgG (Biorad \#1706516, dilution 1:3000) and washed twice for 5 minutes and once for 15 minutes in PBS-Tween. The signal was revealed by enhanced chemiluminescence (ECL) (Millipore) using a PXi-4 gel imaging systems (Syngene).

\section{Results}

Antibodies AF291 and AE391 specifically recognize the HA-tagged AlyA protein (Fig. 1). The protein was also detected with an anti-AlyA antibody (MRB376): a band of the correct size $(\sim 19 \mathrm{kDa})$ can be detected on the overexpressing cells, but is absent in non-transfected alyLKO cells. As previously described, MRB376 does not recognize endogenous AlyA (presumably because the endogenous level of this protein is too low). The antibodies also detect several bands unrelated to the HAtagged protein.

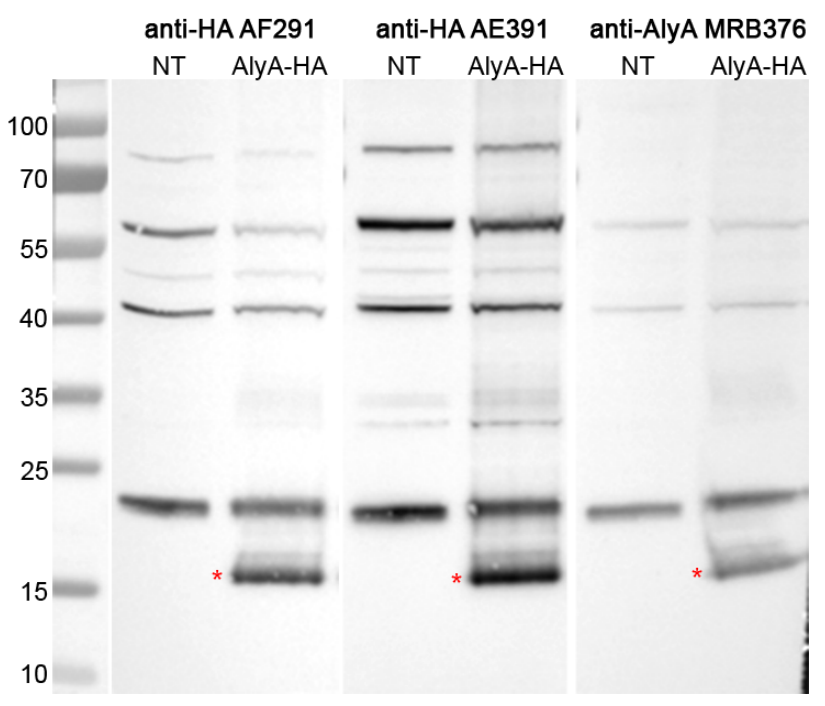

Fig. 1. Specific binding of AF291 and AE391 antibodies to alyL KO cells overexpressing AlyA-HA (position indicated by an asterisk). Nontransfected alyL KO cells (NT) were used as negative control; the endogenous AlyA protein is not detected. MRB376 was used as a positive control for AlyA-HA detection. 


\section{References}

Churchill ME, Stura EA, Pinilla C, et al. Crystal structure of a peptide complex of anti-influenza peptide antibody Fab 26/9. Comparison of two different antibodies bound to the same peptide antigen. J Mol Biol. 1994; 241:53456. PMID:7520084

Keszei Z and Picard D. AF291and AE391 single-chain antibodies recognize the HA tag by Western blotting. Antib. Rep. 2019; 2:e25. doi: 10.24450/journals/abrep.2019.e25

Lamrabet O. RB376 and RB377 antibodies recognize the Dictyostelium AlyA protein by Western blot. Antib. Rep. 2019; 2:e10. doi: 10.24450/journals/abrep.2019.e10

Lima WC, Gasteiger E, Marcatili P, Duek P, Bairoch A, Cosson P. The ABCD database: a repository for chemically defined antibodies. Nucleic Acids Res. 2019; pii:gkz714. PMID:31410491

Niman HL, Houghten RA, Walker LE, et al. Generation of protein-reactive antibodies by short peptides is an event of high frequency: implications for the structural basis of immune recognition. Proc Natl Acad Sci USA. 1983; 80:4949-53. PMID:6192445

\section{Conflict of interest}

The authors declare no conflict of interest. 